\title{
Efectos en el afrontamiento y soporte social ante la revelación de la homosexualidad a la familia: estudio comparativo en gais y lesbianas
}

\section{Effects on coping and social support before the revelation of homosexuality to the family: a comparative study in gays and lesbians}

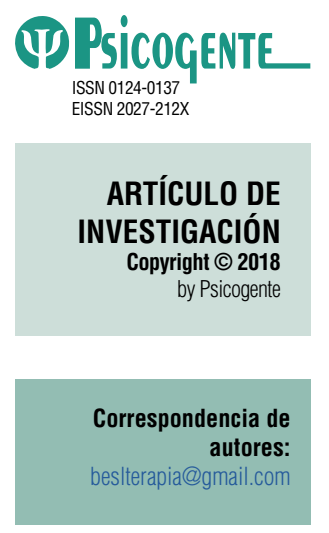

Recibido: 15-03-17 Aceptado: 18-09-17 Publicado: 01-07-18
Benjamín Emanuel Silva Luévanos

Universidad Autónoma de Coahuila, Saltillo, México

Objetivo: El presente trabajo es resultado de una investigación que se realizó en las ciudades de Monclova y Saltillo en el estado de Coahuila, México, con el objetivo de indagar acerca de la autopercepción que tienen gais y lesbianas respecto de los efectos en su afrontamiento y del soporte social después de haber revelado su homosexualidad a la familia.

Método: Para medir las variables de estudio se empleó la Batería de Evaluación Psicosocial de Sucesos Vital (Cantú y Álvarez, 2009; 2013), la cual se aplicó a 30 sujetos: 15 gais y 15 lesbianas. Los datos se analizaron de forma descriptiva y comparando ambos grupos respecto a cada variable, a través de la prueba T de Student.

Resultados: Respecto al afrontamiento y al soporte social no se encuentra diferencia significativa, mostrando ambos grupos estrategias de afrontamiento para resolver problemas. En cuanto al soporte social se encontraron diferencias significativas en dos ítems: "obtuve apoyo emocional de familiares que no viven en casa", y "tuve ayuda económica o de información de mi pareja en relación a la situación", y tanto lesbianas como gais encuentran mayor soporte social en amistades.

Conclusiones: Hombres y mujeres tienen una consideración negativa de la homosexualidad, incluso aquellos que se reconocen como homosexuales; además, tanto gais como lesbianas encuentran mayor soporte fuera de la familia y concretamente en amistades. Por ello, en un inicio la revelación de la propia homosexualidad no suelen hacerla a la familia. Resulta necesario un proceso de reorganización del individuo, así como de la familia y del grupo social a fin de modificar la consideración negativa a priori dominante acerca de la homosexualidad.

Palabras Clave: gay, lesbiana, revelación, familia, afrontamiento, soporte social.

Abstract

Objective: This paper is the result of a research whose purpose is related to gays and lesbian's self-perception and their coping effects and social support after having revealed their homosexuality to their families.

Method: Variables such as Psychosocial Assessment of Vital Events Battery (Cantú y Álvarez, 2009; 2013) was used to measure these variables. 30 individuals were sampled as follows: 15 gays and 15 lesbians. The data analysis was descriptive, where both groups for each variable were compared; Student's T test was used.

Results: In relation with gays and lesbians' coping support, there is no significant differences, in the manner that show coping strategies to solve a situation which they consider a problem; also, in relation with social support, significant differences were found, both lesbians and gays find greater social support in friendships but not from their family, as follows : they got emotional support from family members who do not live with them; and they got financial aid and information help from their couples.

Cómo citar este artículo (APA):

Silva Luévanos, B. E. (2018). Efectos en el afrontamiento y soporte social ante la revelación de la homosexualidad a la familia: estudio comparativo en gais y lesbianas. Psicogente 21(40), 321-336. https://doi.org/10.17081/psico.21.40.3077 
Conclusions: Men and women have a negative opinion related to homosexuality, even those who are recognized as homosexual individual; both gays and lesbians receive more support from people outside the family that means friends. It is necessary to modify the negative and a priori concept of homo-sexuality considered by the society, that is why an individual, family and social group's re-organization process is required.

Keywords: gay, lesbian, revelation, family, coping, social support.

\section{INTRODUCCIÓN}

Constituirse en grupos, es parte y acaso necesidad, de la naturaleza humana. El grupo familiar, por ejemplo, brinda seguridad y protección al individuo, al indicarle normas y conductas que le permiten garantizar la integridad física y emocional. Por tanto, aunque esto se haya afirmado mil veces, hay que reconocer aún que la familia es un pilar fundamental en la formación de individuos, para afrontar los desafíos que implica subsistir en la compleja red de sistemas y organizaciones del mundo.

Ahora bien, el funcionamiento familiar se caracteriza principalmente por la libertad que muestran los miembros para comunicar lo que sienten clara y directamente (Guadarrama y otros, 2011; Sánchez, 2012). Y para las familias de nuestro país, México, la orientación heterosexual de los hijos e hijas no requiere redefinir la relación familiar, puesto que tal orientación constituye una identidad sexual legitimada socialmente. Pero no ocurre lo mismo en el caso de las hijas e hijos que manifiestan una identidad homosexual, pues esta situación obliga a las figuras paterna y materna a asumir un proceso de reconstrucción de dicha relación, de los roles asignados culturalmente y de las expectativas que los padres tenían con respecto a sus hijos (Arévalo y otros, 2011).

Y es que, como institución social, históricamente la familia ha sido la guardiana de las normas de la sexualidad que aseguran la heteronormatividad, y tiende a suprimir a los disidentes de esa norma por medio de la denominada homofobia, que oscurece aún más por la intimidad del mundo privado. Pero ello también trasciende el grupo familiar y potencializa la discriminación y el daño en los espacios sociales (Gonzalez y Silva, 2013).

Según Barrientos y Cárdenas (2010), la cultura que envuelve a estas familias, es igualmente heteronormativa y homofóbica, manifestándose en aspectos como: autoritarismo, conservadurismo valórico, conservadurismo político, roles de género tradicionales, sexismo, religiosidad y actitud negativa hacia el feminismo (Jeremy y otros, 2013; Moral, 2011). 
De esta manera, la homosexualidad de un hijo o hija trunca el equilibrio de la estructura familiar, y genera en sus padres y madres sentimientos de pérdida, conjugados con sensaciones de culpabilidad y negación, sin olvidar el miedo de proyectar el futuro de sus hijos e hijas, que alteran el patrón esperado de la obligatoriedad heterosexual.

En este contexto, la gestión de la homosexualidad de los hijos e hijas es compleja para los padres y madres, pero igualmente complicada para las personas homosexuales que enfrentan directamente la experiencia de disgregación y transgresión social en un sistema heterosexual (Ceballos-Fernández, 2014), y también comprende los sentimientos de miedo a perder el apoyo y confianza familiares (Oksal, 2008).

Por ello, este trabajo busca indagar los efectos de la revelación de la orientación homosexual a la familia, ya que, según Ceballos-Fernández (2014), actualmente este proceso resulta arduo y doloroso, debido a la falta de referentes, desconocimiento, sentimientos de diferencia identitarios y reparos al hecho de confesar la homosexualidad; incluso la mayoría de las personas gais y lesbianas reconocen su miedo a dañar la relación familiar al momento de revelar su orientación sexual (Potoczniak, Crosbie-Burnett, \& Saltzburg, 2009).

Pero antes de continuar en concreto con la investigación, conviene reconocer algunos conceptos claves para la comprensión del asunto: autopercepción, afrontamiento y soporte social, revelación y respuesta, que puede ser negativa o positiva.

\subsection{Autopercepción}

Para el presente trabajo, cobra relevancia el constructo de autopercepción, el cual reúne a su vez conceptos como: actitudes, deseos, expectativas, voliciones y valoraciones, formando con ello un sistema mediante el cual la persona se define a sí misma. Tal autopercepción sumada a la realidad configura la autopercepción social, es decir, la forma en que la persona se ve a sí misma en el mundo e intenta comprenderse mediante un proceso de observación de la propia conducta y las circunstancias que le rodean (Bem, 1967, 1972, citado por Martínez, 2009; Hogg y otros, 2010). En tal sentido, además de requerir un autorreconocimiento y una autoaceptación, las y los homosexuales están claramente mediatizados por la cadena de espacios en los que desarrollan y vivencian su homosexualidad, constituyendo la familia el rol principal de este escenario (Ceballos-Fernández, 2014). 
Dado que la familia es el espacio social donde el ser humano encuentra los primeros y principales recursos para desarrollar su futura personalidad, esta se concibe también como el mayor factor de protección para el individuo siempre que actúe adecuadamente; de lo contrario, se puede convertir en uno de sus mayores riesgos. De esta forma queda claro que la familia juega un papel relevante en la formación de la identidad de cada individuo y en este la orientación sexual es un aspecto que lo configura (Lujan \& Tamarin, 2012).

De acuerdo con las interconexiones que se den en la familia, sus miembros podrán o no afrontar problemas, expresar emociones, manejar las reglas de convivencia y adaptarse a las situaciones de cambio (Rivera, 1999), y es precisamente en la familia, principal medio de socialización y desarrollo para el ser humano, donde las lesbianas y gais enfrentan los estigmas y prejuicios de forma más significativa, lo que impide el proceso de desarrollo de la orientación sexual (Muñoz, Basurod \& Barragan, 2014). Casas (2014) afirma que el rechazo parental al hijo/hija homosexual es considerablemente alto, y puntualiza que por parte de los padres hay en específico mayor actitud negativa, alto sentimiento de deshonra, consideración desagradable de la orientación homosexual y rechazo del lazo matrimonial entre personas del mismo sexo.

\subsection{Afrontamiento y soporte social}

De acuerdo con Macías y otros (2013), las estrategias de afrontamiento son entendidas como recursos psicológicos que el sujeto pone en marcha para hacer frente a situaciones estresantes. Aunque la puesta en marcha de estas no siempre garantizan el éxito, sirven para generar, evitar o disminuir conflictos en los seres humanos, atribuyéndoles beneficios personales y contribuyendo a su fortalecimiento. Además, dichas estrategias no solo se presentan de forma individual, sino que aparecen también como mediadores en el ámbito social.

Uno de los modelos teóricos sobre afrontamiento más citados es el de Lazarus y Folkman (citado en Viñas y otros, 2015), quienes conceptualizan el afrontamiento como los esfuerzos cognitivos y conductuales constantemente cambiantes que sirven para manejar las demandas externas y/o internas que son valoradas como excedentes o desbordantes de los recursos del individuo. Estos mismos autores diferenciaron dos estilos de afrontamiento: el focalizado en el problema o dirigido a su resolución, y el focalizado en las emociones u orientado a restablecer el equilibrio emocional. 
En la medida que la red social, conjunto de lazos relativos a alguna clase de individuos, le proporciona asistencia técnica y tangible a estos, apoyo emocional, sentimientos de cuidado y autoestima, etc., dicha red social le proporciona soporte social (Lemos y Fernández, 1990).

El soporte social puede ser definido, de acuerdo con Cobb (1976, citado por Nunes y otros, 2012), como información que conduce al individuo a creer que es cuidado, amado, estimado y pertenece a una red social con obligaciones mutuas. En otras palabras, el soporte social se refiere a la funcionalidad y calidad de las relaciones interpersonales, así como a la percepción de disponibilidad de ayuda o al verdadero soporte recibido.

Para Sarason, Levine, Basham y Sarason (1983, citados por Nunes y otros, 2012), el soporte social puede ser definido como la existencia o disponibilidad de personas en quienes se puede confiar, ya que se muestran preocupadas por el individuo, lo valoran y le demuestran aprecio. Existen diferentes tipos de apoyo que una persona puede recibir de la red social, siendo tres los más ampliamente estudiados: soporte emocional (percibido como expresión de cariño, cuidados y preocupación), soporte instrumental (ayudas prácticas que puede recibir) y soporte informacional (nociones indispensables que permiten que el individuo oriente en forma adecuada sus acciones al momento de resolver problemas o tomar decisiones).

\subsection{Revelación}

Entre las personas que se autoperciben como gais o lesbianas, un momento crucial en la conformación de la identidad es el de la decisión sobre si han de compartirlo o callarlo (Weststrate \& McLean, 2010). Al respecto, González y Toro (2012) afirman que los gais y lesbianas afirman sentir miedo, frustración y tristeza por lo que dirían otras personas, y en particular sus familiares más cercanos, al enterarse de que son homosexuales, resaltando el dolor que su decisión de aceptar la homosexualidad podría causarles; sin embargo, la mayoría indica que uno de los aspectos que más importancia tuvo en el proceso de aceptación es que otras personas conozcan su orientación homosexual, en particular sus familiares.

Compartir la propia orientación sexual es algo culturalmente complejo para los homosexuales, ya que, como señala Montoya (2009), a los latinos en general se les enseña a buscar ante un conflicto la guía de un sacerdote, o un miembro de la familia y en ningún caso hablar a los "extraños", por lo que el 
rechazo que experimenta un hombre gay latino por parte de los miembros de la familia lo afecta negativamente: la vergüenza, la culpa y el sufrimiento experimentados por este grupo de hombres contribuye a incrementar los ya altos índices de depresión, aislamiento y pensamientos de suicidio.

En este panorama, las personas gais y lesbianas manifiestan al menos tres razones para revelar su orientación sexual a sus padres: 1) reforzar su propia orientación sexual, 2) aclarar aspectos de su identidad y 3) proporcionar información acerca de su estilo de vida. Incluso, las personas que recibieron una respuesta negativa por parte de sus padres, sienten la necesidad de realizar una segunda salida del llamado clóset por los mismos motivos (Denes \& Afifi, 2014).

Ciertamente, la revelación de la homosexualidad del hijo o la hija produce cambios en la relación familiar. Luego de ella se espera una mayor comunicación entre actores y desinhibición al confiarles aspectos íntimos, lo que redundaría en una mayor confianza; sin embargo, los padres reportan vivir este momento con mucho dolor porque no estaba dentro de sus expectativas que su hijo/a tuviera una orientación homosexual, y porque perciben el sufrimiento que ha significado guardar este secreto ante personas tan significativas como lo son las figuras parentales. Reconocen también no sentirse preparados para asumir la homosexualidad de un hijo, puesto que asocian la identidad homosexual con una serie de atributos y estereotipos, lo que urge a una reconstrucción de tal universo simbólico (Arévalo y otros, 2011).

Así, debido a que el sistema familiar no propicia la confianza, la seguridad y el apoyo necesarios que instaría a los sujetos a experimentar su sexualidad y a construir su identidad psicosexual y aceptación explícita al amparo de su familia, los progenitores con hijos e hijas homosexuales requieren orientación y conocimientos, es decir, formarse en el desarrollo de habilidades y competencias psicológicas, educativas, comunicativas y sociales que estimulen el desarrollo de sus hijos e hijas. Y es que las respuestas de los padres a los jóvenes que manifiestan su homosexualidad son cruciales para la posterior adaptación social, puesto que marcará sus experiencias y transiciones personales, de modo que la familia podrá constituir un factor de riesgo perturbador o un elemento beneficioso facilitador en este proceso (Ceballos-Fernández, 2014), toda vez que la divulgación de la propia homosexualidad y el nivel en el desarrollo de la identidad homosexual son elementos intrínsecamente relacionados (Grey y otros, 2013). 


\subsection{Respuesta negativa a la revelación}

El rechazo familiar puede llevar al afectado al consumo de drogas ilegales, depresión, intento de suicidio, así como al riesgo para la salud sexual. En este sentido, tal rechazo es predictor de problemas de salud física y mental. No obstante, una actitud negativa de rechazo puede transformarse positivamente con el tiempo, una vez que los padres y madres se sensibilizan, aprenden y desmitifican lo referente a la homosexualidad de su hijo o hija, mejorando la actitud inicial (Ryan, Russell, Huebner, Diaz \& Sanchez, 2010).

Cuando se percibe un ambiente de rechazo por parte de los grupos de apoyo, las personas gais y lesbianas afrontan la incapacidad de compartir e internalizan este rechazo, que se manifiesta con conductas autodestructivas como las antes descritas. Además, como lo señalan Fernández y Vázquez (2013), al estar expuestos al rechazo desde la infancia, hace que la persona asuma como natural la homofobia, por lo que se vive una identidad homosexual desintegrada. Superar tal homofobia internalizada supone el apoyo social de la familia, la escuela, los amigos y la comunidad.

El rechazo a sí mismo o, con otras palabras, la homofobia o el autoestigma es indicador de baja autoestima, poca claridad de autoconcepto y mayor confusión de identidad sexual, que se asocian a síntomas depresivos, de acuerdo con lo reportado en su estudio por Feinstein, Davila y Yoneda (2011), así como a conductas autodestructivas manifiestas en el uso y abuso de drogas (Rosario, Schirimshaw \& Hunter, 2009).

Cobra especial relevancia, entonces, una actitud negativa por el daño que puede causar, y es importante entender que dicha actitud está relacionada con el conservadurismo de los padres, valores tradicionales, fuertes creencias religiosas, la rigidez en el ejercicio de la autoridad y las ideas erróneas sobre la homosexualidad, así como con recursos familiares pobres para hacer frente a una situación de estrés (Baiocco y otros, 2014).

\subsection{Respuesta positiva a la revelación}

Como se ha afirmado, compartir con la familia es de suma importancia y en especial porque la aceptación de la familia se asocia de manera positiva con la salud mental y física, expresados en el buen ajuste psicológico y la evitación del abuso en el consumo de drogas y alcohol (Ryan, Russell, Huebner, Diaz, y Sanchez, J. 2010; D’Amico \& Julien, 2012). 
Una identidad integrada se relaciona con menos síntomas depresivos y ansiosos, menos problemas de conducta y mayor autoestima. Es claro al respecto que los padres tienen una tendencia a rechazar o medianamente aceptar a los hijos e hijas cuando les comparten su orientación homosexual, pero ello puede mejorar y redundar a una mayor aceptación a partir del compromiso como padres, el contacto con personas gais y lesbianas informadas y pláticas con su hijo o hijas. Por ello la formación familiar sobre el tema es potencialmente positiva en la aceptación y la subsecuente conformación de la identidad homosexual (Rosario, Schirimshaw \& Hunter 2009; Samarova, Shilo, \& Diamond, 2013)

\section{MÉTODO}

\subsection{Diseño}

El artículo presente es resultado de un estudio cuantitativo, elaborado con un diseño no experimental, exploratorio y transeccional (García, 2009).

\subsection{Instrumentos}

Se utilizaron dos escalas tipo Likert de la Batería de Evaluación Psicosocial de Suceso Vital (Cantú y Álvarez, 2009; 2013), cada una con una confiabilidad de .70: Escala de Afrontamiento Respecto de un suceso Vital, 21 items, una dimensión; y Escala de Soporte Social Respecto de un Suceso Vital, 27 items, una dimensión.

\subsection{Participantes}

En el estudio participaron 15 hombres autorreconocidos como gais y 15 mujeres auto-reconocidas como lesbianas, de entre 18 y 60 años, que habitan en las ciudades de Saltillo y Coahuila, en el estado de Coahuila, México.

\subsubsection{Criterios de inclusión y exclusión}

Para la muestra se consideraron sujetos gais y lesbianas auto-reconocidos, que habitan en el estado de Coahuila y con mayoría de edad legal mínima, 18 años. Se excluyeron las personas que habitan en otros estados y que no cumplen con la mayoría de edad.

\subsection{Procedimiento}

Se contactó a las personas gais y lesbianas conocidas, se les presentó el proyecto del trabajo y se solicitó su participación. A quienes aceptaron, se les pidió que recomendaran a otras personas que tuvieran rasgos de interés 
similares para tomar datos, como una cadena. Ferrel y otros (2013) afirman que la población de hombres exclusivamente homosexuales van del $4 \%$ al 17 $\%$, lo que complejiza la selección de la muestra para estudios estadísticos, por lo que se optó por la técnica de bola de nieve para la selección de sus sujetos, no solo por el porcentaje antes señalado sino por el desconocimiento de la población total.

Luego de observar a los sujetos designados, se siguió de la misma manera hasta obtener el número suficiente de sujetos. La aplicación de instrumentos y entrevista se efectuó en una sola exhibición.

Martínez-Salgado (2012) aseguran que en casos como estos no hay reglas para decidir el tamaño de la muestra y, si hubiera que enunciar alguna, esta sería: "todo depende". Depende del propósito del estudio, de lo que resulta útil para lograrlo, de lo que está en juego, de lo que lo hace verosímil y, en última instancia, de lo que es posible. Así, para juzgar si una muestra es adecuada, hay que conocer el contexto del estudio.

El precepto exige recolectar datos hasta que ocurra la saturación, es decir, cuando se entiende que ha llegado el punto en el cual se ha escuchado ya una cierta diversidad de ideas y con cada entrevista u observación adicional no aparecen ya otros elementos. Mientras sigan apareciendo nuevos datos o nuevas ideas, la búsqueda no debe detenerse.

\subsection{Análisis de datos}

El análisis de los datos se realizó con el paquete estadístico SPSS, mediante la prueba T de Student para muestras independientes.

\subsection{Conflicto de interés}

La investigación no se relaciona con ninguna institución, por lo que los resultados no son particularmente convenientes para institución alguna.

\section{RESULTADOS}

Se compararon las medias mediante la prueba T de Student (sig. > 0.05) con el paquete estadístico SPSS.

No se encontró diferencia significativa en el afrontamiento de la identidad en gais y lesbianas (Tabla 1), siendo las más altas estrategias: "Acepté que tengo una situación que antes no tenía", "Hable con mis amigos y amigas de cómo me sentí en relación a mi situación gay/lésbica", "Negué que viví la situación" y "Busqué personas que me puedan ayudar a manejar la situación". 


\section{Tabla 1}

Resultados en la Escala de Afrontamiento respecto de un suceso vital (21 items)

\begin{tabular}{|c|c|c|c|c|}
\hline COMPONENTES SUCESO VITAL & O. SEXUAL & $\mathbf{N}$ & MEDIA & SIG. \\
\hline \multirow[t]{2}{*}{1 Negué que viví la situación } & Gay & 15 & 3.13 & \\
\hline & Lesbiana & 15 & 2.87 & 0.626 \\
\hline \multirow[t]{2}{*}{2 Intenté informarme acerca de mi situación } & Gay & 15 & 2.73 & \\
\hline & Lesbiana & 15 & 2.47 & 0.629 \\
\hline \multirow[t]{2}{*}{3 Luché lo más que pude contra ella } & Gay & 15 & 2.67 & \\
\hline & Lesbiana & 15 & 2.47 & 0.718 \\
\hline \multirow[t]{2}{*}{4 Hice un gran esfuerzo para olvidarme que estoy enfermo } & Gay & 15 & 2.47 & \\
\hline & Lesbiana & 15 & 2.07 & 0.496 \\
\hline \multirow[t]{2}{*}{5 Busqué apoyo emocional de mis padres en relación a mi situación } & Gay & 15 & 2.6 & \\
\hline & Lesbiana & 15 & 1.73 & 0.396 \\
\hline \multirow[t]{2}{*}{6 Busqué atención profesional médica y sigo al pie de la letra los tratamientos médicos } & Gay & 15 & 1.53 & \\
\hline & Lesbiana & 15 & 1.53 & 1 \\
\hline \multirow[t]{2}{*}{7 Reclamé mi enojo a las personas responsables de mis problemas } & Gay & 15 & 1.6 & 0.116 \\
\hline & Lesbiana & 15 & 3.2 & \\
\hline \multirow[t]{2}{*}{8 Acepté que tengo una situación que antes no tenía } & Gay & 15 & 2.87 & \\
\hline & Lesbiana & 15 & 3.67 & 0.143 \\
\hline \multirow[t]{2}{*}{9 Busqué apoyo emocional en familiares que viven en casa en relación a mi situación } & Gay & 15 & 1.73 & \\
\hline & Lesbiana & 15 & 2.27 & 0.349 \\
\hline \multirow[t]{2}{*}{10 Busqué personas que me puedan ayudar a manejar la situación } & Gay & 15 & 2.87 & \\
\hline & Lesbiana & 15 & 2.87 & 1 \\
\hline \multirow[t]{2}{*}{11 Busqué apoyo emocional en familiares que no viven en casa en relación a mi situación } & Gay & 15 & 1.47 & \\
\hline & Lesbiana & 15 & 1.33 & 0.206 \\
\hline \multirow[t]{2}{*}{12 Tomé alcohol u otras sustancias para olvidar los problemas relacionados con la situación } & Gay & 15 & 1.13 & \\
\hline & Lesbiana & 15 & 2.27 & 0.128 \\
\hline \multirow[t]{2}{*}{13 Busqué ayuda espiritual en relación a mi situación } & Gay & 15 & 2.4 & \\
\hline & Lesbiana & 15 & 1.8 & 0.286 \\
\hline \multirow[t]{2}{*}{14 Trabajé mucho para olvidar los problemas relacionados a la situación } & Gay & 15 & 2.2 & \\
\hline & Lesbiana & 15 & 2.33 & 0.79 \\
\hline \multirow[t]{2}{*}{15 Busqué atención profesional psicológica en relación a mi situación } & Gay & 15 & 2.13 & \\
\hline & Lesbiana & 15 & 1.73 & 0.413 \\
\hline \multirow[t]{2}{*}{16 Intenté mantenerme ocupado para no pensar en mi situación } & Gay & 15 & 2.33 & \\
\hline & Lesbiana & 15 & 2.6 & 0.619 \\
\hline \multirow[t]{2}{*}{17 Busqué apoyo emocional en mi pareja en relación a mi situación } & Gay & 15 & 1.4 & \\
\hline & Lesbiana & 15 & 2.53 & 0.176 \\
\hline \multirow[t]{2}{*}{18 Creía que mi situación es debido a un error por parte de los involucrados } & Gay & 15 & 1.8 & \\
\hline & Lesbiana & 15 & 1.53 & 0.582 \\
\hline \multirow[t]{2}{*}{$\begin{array}{l}19 \text { A partir de darme cuenta de mi situación cuidé más mis hábitos de salud, descanso, sueño, } \\
\text { alimentos, otros }\end{array}$} & Gay & 14 & 2.21 & \\
\hline & Lesbiana & 15 & 1.8 & 0.418 \\
\hline \multirow[t]{2}{*}{20 Hablé con mis amigos y amigas de cómo me sentí en relación a mi situación gay/lésbica } & Gay & 15 & 3.2 & \\
\hline & Lesbiana & 15 & 3.13 & 0.915 \\
\hline \multirow[t]{2}{*}{21 Intenté no hablar y que no me hablaran de mi situación } & Gay & 15 & 2 & \\
\hline & Lesbiana & 15 & 1.87 & 0.777 \\
\hline
\end{tabular}


En cuanto al Soporte Social (Tabla 2), no se presentaron diferencias significativas entre los items con medias con mayor puntaje: "Percibí que cuento con ayuda de parte de mis amistades en relación a la situación", "Sentía que contaba con personas que se preocupan de mis problemas", "Percibí apoyo de la gente que me rodea", "Hablé con alguien de mis problemas en general y sobre cómo me siento".

Tabla 2

Resultados en la Escala de Soporte Social respecto de un suceso vital (27 items)

\begin{tabular}{|c|c|c|c|c|}
\hline SUCESO VITAL (2) & O. SEXUAL & $\mathbf{N}$ & MEDIA & SIG. \\
\hline & Lesbiana & 15 & 3.13 & 0.409 \\
\hline \multirow[t]{2}{*}{2 Obtuve apoyo emocional de mi madre en relación a mi situación } & Gay & 15 & 2.73 & \\
\hline & Lesbiana & 15 & 2.6 & 0.846 \\
\hline \multirow[t]{2}{*}{3 Tuve apoyo de orientación o información de mi madre en relación a mi situación } & Gay & 15 & 2 & \\
\hline & Lesbiana & 15 & 1.73 & 0.551 \\
\hline \multirow[t]{2}{*}{4 Obtuve apoyo de amistades en relación a mi situación } & Gay & 15 & 2.87 & \\
\hline & Lesbiana & 15 & 3.13 & 0.637 \\
\hline \multirow[t]{2}{*}{5 Recibí apoyo médico o psicológico en relación a la situación } & Gay & 15 & 1.53 & \\
\hline & Lesbiana & 15 & 1.67 & 0.747 \\
\hline \multirow[t]{2}{*}{6 Obtuve apoyo emocional de mi padre en relación a la situación } & Gay & 15 & 1.4 & \\
\hline & Lesbiana & 15 & 1.87 & 0.314 \\
\hline \multirow[t]{2}{*}{7 Hablé con alguien de mis problemas en general y sobre cómo me siento } & Gay & 15 & 2.93 & \\
\hline & Lesbiana & 15 & 3.2 & 0.646 \\
\hline \multirow[t]{2}{*}{8 Percibí apoyo de mi madre en relación a la situación de ser gay o lesbiana } & Gay & 15 & 3 & \\
\hline & Lesbiana & 15 & 2.8 & 0.777 \\
\hline \multirow[t]{2}{*}{9 Obtengo apoyo económico de mi padre en relación a la situación de ser gay o lesbiana } & Gay & 15 & 2 & \\
\hline & Lesbiana & 15 & 2.67 & 0.275 \\
\hline \multirow[t]{2}{*}{10 Percibí apoyo de la gente que me rodea } & Gay & 15 & 3.33 & \\
\hline & Lesbiana & 15 & 3.4 & 0.904 \\
\hline \multirow[t]{2}{*}{11 Obtuve ayuda económica por mi situación de familiares que no viven en casa } & Gay & 15 & 1.4 & \\
\hline & Lesbiana & 15 & 1.33 & 0.848 \\
\hline \multirow[t]{2}{*}{12 Percibí que cuento con ayuda de parte de mi padre en relación a la situación } & Gay & 15 & 1.73 & \\
\hline & Lesbiana & 15 & 2.27 & 0.283 \\
\hline \multirow[t]{2}{*}{13 Tuve ayuda económica por mi situación de familiares que viven en casa } & Gay & 15 & 1.87 & \\
\hline & Lesbiana & 15 & 1.93 & 0.893 \\
\hline \multirow[t]{2}{*}{14 Percibí apoyo de otros familiares que viven en casa en relación a la situación } & Gay & 15 & 1.93 & \\
\hline & Lesbiana & 15 & 2.4 & 0.392 \\
\hline \multirow[t]{2}{*}{15 Obtuve apoyo de familiares que no viven en casa en relación a la situación } & Gay & 15 & 2.27 & \\
\hline & Lesbiana & 15 & 2.73 & 0.575 \\
\hline \multirow[t]{2}{*}{16 Percibí apoyo de familiares que no viven en casa en relación a la situación } & Gay & 15 & 1.8 & \\
\hline & Lesbiana & 15 & 2.73 & 0.08 \\
\hline
\end{tabular}




\begin{tabular}{|c|c|c|c|c|}
\hline SUCESO VITAL (2) & O. SEXUAL & $\mathbf{N}$ & MEDIA & SIG. \\
\hline \multirow[t]{2}{*}{17 Obtuve apoyo de mi madre en relación a la situación } & Gay & 15 & 2.53 & \\
\hline & Lesbiana & 15 & 2.53 & 1 \\
\hline \multirow[t]{2}{*}{18 Obtuve apoyo emocional de otros familiares que viven en casa en relación a la situación } & Gay & 15 & 2.4 & \\
\hline & Lesbiana & 15 & 2.33 & 0.914 \\
\hline \multirow[t]{2}{*}{19 Obtuve apoyo de información o económico de mi padre en relación a la situación } & Gay & 15 & 1.6 & \\
\hline & Lesbiana & 15 & 1.93 & 0.475 \\
\hline \multirow[t]{2}{*}{20 Obtuve apoyo de otros familiares que viven en casa en relación a la situación } & Gay & 15 & 1.93 & \\
\hline & Lesbiana & 15 & 2.33 & 0.469 \\
\hline \multirow[t]{2}{*}{21 Obtuve apoyo emocional de familiares que no viven en casa en relación a la situación } & Gay & 15 & 1.4 & \\
\hline & Lesbiana & 15 & 2.87 & 0.001 \\
\hline \multirow[t]{2}{*}{22 Percibí que cuento con ayuda de parte de mis amistades en relación a la situación } & Gay & 15 & 3.27 & \\
\hline & Lesbiana & 15 & 3.67 & 0.469 \\
\hline \multirow[t]{2}{*}{23 Tuve ayuda económica o de información de mi pareja en relación a la situación } & Gay & 15 & 1.27 & \\
\hline & Lesbiana & 15 & 2.4 & 0.023 \\
\hline \multirow[t]{2}{*}{24 Obtuve apoyo emocional de mi pareja o esposo(a) en relación a la situación } & Gay & 15 & 1.87 & \\
\hline & Lesbiana & 15 & 2.47 & 0.306 \\
\hline \multirow[t]{2}{*}{25 Conté con personas que se preocupan de mis problemas } & Gay & 15 & 3.4 & \\
\hline & Lesbiana & 15 & 3.47 & 0.906 \\
\hline \multirow[t]{2}{*}{26 Recibí apoyo emocional del médico o psicologo en relación a mi situación } & Gay & 15 & 1.67 & \\
\hline & Lesbiana & 15 & 1.6 & 0.868 \\
\hline \multirow[t]{2}{*}{27 Obtuve apoyo económico o de información de mi situación de un grupo de ayuda } & Gay & 15 & 1.47 & \\
\hline & Lesbiana & 15 & 1.07 & 0.19 \\
\hline
\end{tabular}

\section{DISCUSIÓN}

Al no encontrar diferencias significativas en el afrontamiento de gais $y$ lesbianas, en cuanto a los recursos psicológicos que los dos grupos ponen en marcha para hacer frente a la situación de ser homosexuales y revelarlo, es posible afirmar, de acuerdo con Lazarus y Folkman, que tales estrategias parecieran focalizarse en la resolución del "problema" para restablecer el propio equilibrio emocional.

En cuanto al soporte social, se encontraron diferencias significativas en los ítems: "Obtuve apoyo emocional de familiares que no viven en casa en relación a la situación", y "Tuve ayuda económica o de información de mi pareja en relación a la situación", siendo en ambos casos las lesbianas quienes reportan un mayor puntaje. Siguiendo a Lemos y Fernández (1990), las lesbianas cuentan con mayores lazos que proporcionan soporte social y, de acuerdo con Sarason (1983, citado por Nunes, 2012), ellas contarían con 
más personas en quienes se puede confiar, que se muestran preocupadas por ellas y que las valoran y les demuestran aprecio.

También debe considerarse que el mayor puntaje en las medias para ambos grupos indica que tal soporte social se encuentra en las amistades. Como señala Fernández y Vázquez (2013), tanto gais como lesbianas han sido expuestos en sus familias a un ambiente de rechazo hacia la homosexualidad, por lo que revelarles su orientación sexual a estos no resulta una opción primaria, antes bien parece confirmarse lo que señalan las investigaciones de Oksal (2008) y González y Toro (2012): en estos grupos hay un temor a perder el apoyo familiar.

\section{CONCLUSIÓN}

Las personas gais y lesbianas afrontan en un inicio su homosexualidad como un problema a resolver, es decir, hombres y mujeres tienen una consideración negativa de la homosexualidad, incluso aquellos que se reconocen como homosexuales; esto por el enorme contexto de homofobia social y familiar en que se desenvuelven. Si bien las lesbianas parecen contar con mayores recursos como soporte social, pues reportan apoyo por parte de personas que no viven en la casa familiar y de la pareja, tanto ellas como los gais encuentran mayor soporte por fuera de la familia y, concretamente, en amistades.

Por lo anterior, es posible afirmar que la revelación de la propia homosexualidad, tanto en gais como en lesbianas no involucra, al menos en un principio, a la familia, pues consideran que serán rechazados principalmente por ella.

Es necesario modificar la consideración negativa a priori sobre la homosexualidad que domina en la sociedad para que el individuo que se descubre homosexual pueda contar con el soporte social familiar y para que sus acciones de afrontamiento conlleven al desarrollo y no a la resolución, de donde resulta necesario un proceso de reorganización del individuo, de la familia y del grupo social.

Nota de autores:

El presente trabajo forma parte de la investigación "Las representaciones sociales en el desarrollo de la identidad sexual de gais y lesbianas".

\section{REFERENCIAS}

Arévalo, M., Lizama, C. \& Sanhueza, L. (2011). Revelación de homosexualidad de un hijo y reconstrucción del vínculo parento-filial. Perspectivas, 14(22), 105-118. 
Recuperado de http://ediciones.ucsh.cl/ojs/index.php?journal=Perspectivas\&page=article\&op=view \&path\%5B\%5D=570\&path\%5B\%5D =455

Baiocco, R., Fontanesi, L., Santamaria, F., loverno, S., Marasco, B., Baumgartner, E., Willoughby, B.L.B. \& Laghi, F. (2014). Negative Parental Responses to Coming Out and Family Functioning in a Sample of Lesbian and Gay Young Adults. Journal of Child and Family Studies, 24(5), 1490-1500. https://doi.org/10.1007/s10826014-9954-z

Barrientos, J. y Cárdenas, J. (2010). Adaptación y validación de la escala Likert de actitudes de heterosexuales hacia homosexuales (HATH) en una muestra de estudiantes universitarios chilenos. Revista Latinoamericana. (5), 30-49. Recuperado de http://www.redalyc.org/articulo.oa?id=293323015003

Cantú, G. R. \& Álvarez, B. J. (2009). Sucesos vitales y factores psicosociales asociados: el caso de paciente con cáncer. Psicología y Salud, 19 (1), 21-32. Recuperado de http://revistas.uv.mx/index.php/psicysalud/article/view/642/0

Cantú, R. \& Álvarez, J. (2013). Batería de Evaluación psicosocial del suceso de enfermedad crónica: niveles de confiabilidad y estructura factorial. Medicina, Salud y Sociedad, 4 (1), 59-80. http://dx.doi.org/10.25009/remsys.2013.1.58

Casas, D. (2014). Rechazo parental en homosexuales de una unidad de medicina familiar, Revista Brasileira de Medicina de Familia e Comunidade, 9(31) 127-132. http://dx.doi.org/10.5712/rbmfc9(31)547

Ceballos-Fernández, M. (2014). Identidad homosexual y contexto familiar heteroparental: implicaciones educativas para la subversión social. Revista Latinoamericana de Ciencias Sociales, Niñez y Juventud, 12(2), 643-658. Disponible en http://revistaumanizales.cinde.org.co/rlcsnj/index.php/Revista-Latinoamericana/article/view/1365

D’Amico, E. \& Julien, D. (2012). Disclosure of Sexual Orientation and Gay, Lesbian, and Bisexual Youths' Adjustment: Associations with Past and Current Parental Acceptance and Rejection. Journal of GLBT Family Studies, 8(3), 215-242. https:// doi.org/10.1080/1550428X.2012.677232

Denes, A. \& Afifi, T. D. (2014). Coming Out Again: Exploring GLBQ Individuals' Communication with Their Parents After the First Coming Out. Journal of GLBT Family Studies, 10(3), 298-325. https://doi.org/10.1080/1550428X.2013.838150

Feinstein, B., Davila, J. \& Yoneda, A. (2011). Self-concept and self-stigma in lesbian and gay men. Psychology \& Sexuality, 3(2), 161-177. https://doi.org/10.1080/19 419899.2011.592543

Fernández, M. \& Vázquez, F. (2013). En torno al rechazo, la salud mental y la resiliencia en un grupo de jóvenes universitarios gays y lesbianas. Griot, 6(1), 44-65. Disponible en https://www.ncbi.nlm.nih.gov/pmc/articles/PMC4318519/

Ferrel, O. F., González, O. J. \& Padilla, M. Y. (2013) Esquemas mal adaptativos tempranos y creencias en un grupo de homosexuales masculinos de la ciudad de Santa Marta, Colombia. Psicología desde el Cribe, 30(1) 36-66. Disponible en http://rcientificas.uninorte.edu.co/index.php/psicologia/article/view/4447/6900

García C. B. (2009), Manual de métodos de investigación para las ciencias sociales. Un enfoque de enseñanza basado en proyectos. Manual Moderno, Ciudad de México. Disponible en http://www.manualmoderno.com/tienda/index. php/9786074480115.html

Gonzales, L. \& Silva F. (2013). Homofobia familiar: abrindo o armario "entre Quatro paredes". Arquivos Brasileiros de Psicología, 65(3), 375-391. Disponible en http://pepsic.bvsalud.org/scielo.php?script=sci_arttext\&pid=S1809-52672013 000300005 
González, J. \& Toro, J. (2012). El significado de la experiencia de la aceptación de la orientación sexual homosexual desde la memoria de un grupo de hombres adultos puertorriqueños. Psicoeureka, 9(2), 158-170. Recuperado de http://pepsic. bvsalud.org/scielo.php?script=sci_arttext\&pid=S2220-90262012000200004

Grey, A., Robinson, B., Coleman, E. \& Bocking, W. (2013). A Systematic Review of Instruments that measure attitudes toward homosexual men. Journal of sex reserch, 50(3-4), 329-352. https://doi.org/10.1080/00224499.2012.746279

Guadarrama, R., Maruqez, O. \& Veytia M. (2011). Funcionamiento familiar en estudiantes de nivel superior. Revista Electrónica de Psicología Iztacala, 14 (2), 179-192. Recuperado de http://www.journals.unam.mx/index.php/repi/article/ view/26033

Hogg, M-A., Hogg, M-G., Vaughan, G. \& Haro, M. (2010). Psicología Social. Madrid: Ed. Médica Panamericana

Jeremy, A., Grey, B., Robinson, E. \& Walter, B. (2013). A systematic review of instruments that measure attitudes toward homosexual men. The Journal of Sex Research, 50 (3-4), 329-352. https://doi.org/10.1080/00224499.2012.746279

Lemos, G. \& Fernández, H. (1990). Redes de soporte social y salud. Psicothema, 2(2), 113-135. Recuperado de http://www.psicothema.com/psicothema.asp?id=671

Lujan, I \& Tamarin, A. (2012). Dinámica familiar ante la revelación de la orientación homosexual de los hijos/as. International Journal of developmental and educational psychologi, 1(3), 301-308. Recuperado de http://dehesa.unex.es/ bitstream/handle/10662/2903/0214-9877_2012_1_3_309.pdf?sequence=1

Macías, M., Madariaga, O., Valle, A., Zambrano, J. (2013). Estrategias de afrontamiento individual y familiar frente a situaciones de estrés psicológico. Psicología desde el Caribe, 30(1), 123-145. Recuperado de http://rcientificas.uninorte.edu. co/index.php/psicologia/article/view/2051/6906

Martínez, H. (2009). Autopercepción social y atribuciones cognoscitivas en estudiantes de bajo rendimiento académico. Education \& Psychology, 7(3), 1175-1216. Recuperado de http://www.redalyc.org/articulo.oa?id=293121984012

Martínez-Salgado, C. (2012). El muestreo en investigación cualitativa. Principios básicos y algunas controversias. Ciencia \& Sau de Colectiva, 17(3), 613-619. http://dx.doi.org/10.1590/S1413-81232012000300006

Montoya Tajon, M. (2009). Identity development of latino gay men. (Tesis de pregrado). Antioch University, Santa Barbara. Recuperado de http://aura.antioch. edu/etds/128/

Moral de la Rubia, J. \& Valle de la O, A. (2011). Escala de Actitudes hacia Lesbianas y Hombres Homosexuales en México 1. Estructura factorial y consistencia interna. Nova Scientia, 3(6), 139-157. Recuperado de http://www.redalyc.org/articulo. oa? id $=203318388008$

Muñoz, E., Basurod, M. \& Barragán, O. (2014). Construcción de factores protectores en la familia para el afrontamiento de la discriminación en adultos jóvenes homosexuales. (Tesis de grado). Universidad Nacional Abierta y a Distancia, Granada. Recuperado de http://stadium.unad.edu.co/preview/UNAD.php?url=/ bitstream/10596/2438/3/1120364523.pdf

Nunes, B., Rigotto, D., y Ferrari, C. (2012). Soporte social, familiar y autoconcepto: relación entre los constructos. Psicología desde el Caribe, 29(1), 1-18. Recuperado de http://www.scielo.org.co/pdf/psdc/v29n1/v29n1a02.pdf

Oksal, A. (2008). Turkish Family members'Attitudes Toward Lesbian and Gay Men. Sex Roles, 58, 514-525. https://doi.org/10.1007/s11199-007-9370-6 
Potoczniak, D., Crosbie-Burnett, M. \& Saltzburg, N. (2009). Experiences Regarding Coming Out to Parents Among African American, Hispanic, and White Gay, Lesbian, Bisexual, Transgender, and Questioning Adolescents. Journal Of Gay \& Lesbian Social Services, 21(2-3), 189-205. https://doi.org/10.1080/10538720902772063

Rivera, E. (1999). Instrumento de evaluación de las relaciones intrafamiliares en Calleja, N. (comp.) (2011). Inventario de escalas psicosociales en México 1984-2005, México: UNAM. Recuperado de http://www.psicologia.unam.mx/ contenidoEstatico/archivo/files/Investigaci\%C3\%B3n/Nazira\%20Calleja\%20 -Inventario\%20de\%20escalas\%20psicosociales\%20en\%20M\%C3\%A9xico,\%20 1984-2005.pdf

Rosario, M., Schirimshaw, E. \& Hunter J. (2009). Disciosure of sexual orientation and subsequent substance use and abuse among lesbian, gay, and bisexual youths: critical role of disciosure reactions. Psychol Addict Behav, 23(1), 175-184. https:// doi.org/ 10.1037/a0014284

Ryan, C., Russell, S. T., Huebner, D., Diaz, R. \& Sanchez, J. (2010). Family Acceptance in Adolescence and the Health of LGBT Young Adults. Journal of Child and Adolescent Psychiatric Nursing, 23(1), 205-213. https://doi.org/10.1111/j.17446171.2010.00246.x

Samarova, V., Shilo, G. \& Diamond, G. M. (2013). Changes in Youths' Perceived Parental Acceptance of Their Sexual Minority Status over Time. Journal of Research on Adolescence, 24(4), 681-688. https://doi.org/10.1111/jora.12071

Sánchez Martínez, C. (2012). Significado psicológico de la familia, papá y mamá en adolescentes. Psicología iberoamericana, 20(1), 18-28. Recuperado de http:// www.redalyc.org/articulo.oa?id=133924623003

Viñas, F., González, M., García, Y., Malo, S., Casas, F., y Casas, C. (2015). Los estilos y estrategias de afrontamiento y su relación con el bienestar personal en una muestra de adolescentes. Anales de Psicología, 31(1), 226-233. http://dx.doi. org/10.6018/analesps.31.1.163681

Weststrate, N \& McLean K. (2010). The rise and fall of gay: a cultural-historical approach to gay identity development. Psychology Press, 18(2), 225-240. https:// doi.org/10.1080/09658210903153923 autor o el licenciante.

\section{(c))BY}

